\title{
Postponed absorption of dabigatran in a patient with paralytic ileus - difficulties in decision making on reversal agent (idarucizumab) use
}

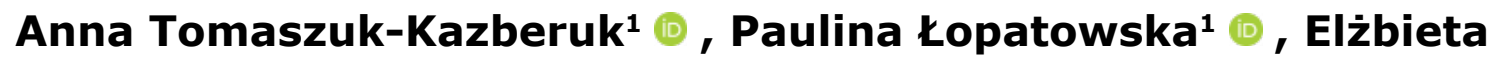 \\ Młodawska1 ${ }^{\circledR}$, Beata Klaja², Paulina Ausztol², Bolesław Korbel ${ }^{3}$, \\ Bożena Sobkowicz ${ }^{1}$ \\ ${ }^{1}$ Department of Cardiology, Medical University of Białystok, Poland \\ ${ }^{2}$ Student Scientific Club at the Department of Cardiology, Medical University of Białystok, Poland \\ ${ }^{3}$ Boehringer Ingelheim, Warszawa, Poland
}

\begin{abstract}
According to the European Society of Cardiology (ESC) guidelines, non-vitamin $\mathrm{K}$ antagonist oral anticoagulants (NOACs) are first choice drugs in prevention of thromboembolic events among patients with atrial fibrillation (AF). According to our knowledge this was the only case of delayed absorption of dabigatran due to ileus. A 79-year-old woman with hypertension and a 1-year history of persistent AF treated with dabigatran (a direct thrombin inhibitor approved for the prevention of stroke in patients with non-valvular AF) $110 \mathrm{mg}$ bid for two weeks. She was hospitalized due to acute abdominal pain, vomiting and diarrhea. Signs of acute embolism of abdominal aorta (paraparesis) were confirmed on contrast-enhanced computed tomography scan and she was qualified for emergency surgery. The use of idarucizumab, the specific reversal agent for dabigatran, was considered twice. This case shows that exposure to dabigatran may occur later in patient with acute ileus due to alterations in absorption than it can be expected in normal situation. Such patients should be carefully monitored for a significant rebound in anticoagulant activity.

Keywords: idarucizumab • dabigatran • paralytic ileus • absorption
\end{abstract}

\section{Citation}

Tomaszuk-Kazberuk A, Łopatowska P, Młodawska E, Klaja B, Ausztol P, Korbel B, Sobkowicz B. Postponed absorption of dabigatran in a patient with paralytic ileus - difficulties in decision making on reversal agent (idarucizumab) use. Eur J TransI Clin Med. 2019;2(1):44-47.

DOI: $10.31373 /$ ejtcm/109298 


\section{Background}

Idarucizumab, a dabigatran reversal agent, is the only antidote for NOACs available in Europe. In May 2018, andexanet alfa an antidote for factor Xa inhibitor (apixaban, rivaroxaban and edoxaban) was approved for sale in the United States of America. Idarucizumab is a humanized monoclonal antibody fragment that binds to dabigatran with a more than 350-fold higher affinity than dabigatran does to thrombin, therefore it instantly, specifically, strongly, and durably binds dabigatran and quickly reverses its anticoagulation effects [1]. The dabigatran-idarucizumab complex is biochemically stable and eliminated by the kidneys within a few hours. Dabigatran therapy may be reinitiated 24 hours after antidote administration. Efficacy and safety of idarucizumab was demonstrated in patients with major bleeding or who required urgent surgery or invasive procedures in the REVERSE-AD study [1]. Adverse reactions were observed in only $\leq 5 \%$ of patients and included headache and hypokalemia. Idarucizumab has been successfully administered in patients with life-threatening bleeding or before urgent major surgeries [2-4].

\section{Aim}

We wanted to analyze if exposure to dabigatran may occur later due to alterations in absorption in patients with acute ileus.

\section{Results}

\section{Series analysis}

A single occurrence of altered absorption of dabigatran triggered the documentation analysis of all patients treated with dabigatran due to AF since January 2017 to December 2018. In this single-centered retrospective study, 387 patients were reviewed for two endpoints: life-threatening bleeding and altered absorption of dabigatran. We recorded only two cases of life-threating bleeding in which idarucizumab was successfully used and one case of altered absorption of dabigatran.

\section{Case description}

A 79-year-old woman with hypertension and a 1-year history of persistent atrial fibrillation (AF) treated with dabigatran (Pradaxa ${ }^{\circledR}$, Boehringer Ingelheim, a direct thrombin inhibitor that is approved for the prevention of stroke in patients with non-valvular AF) $110 \mathrm{mg}$ bid for two weeks, was hospitalized due to acute abdominal pain, vomiting and diarrhea. Upon admission, the patient was dehydrated, with blood pressure of $165 / 65 \mathrm{mmHg}$ and a heart rate of 85 beats/minute. The physical examination revealed abdominal tenderness and positive Blumberg's sign. The patient was given i.v. fluids, spasmolytics, painkillers and her condition markedly improved. Electrocardiogram (ECG) showed AF. Initial laboratory work-up revealed normal kidney function.

On the next day dabigatran was administered at 0800 . Two hours later the patient presented with acute abdominal pain, paralytic ileus and deterioration of general condition. Signs of acute embolism of abdominal aorta (paraparesis) were confirmed on contrast-enhanced computed tomography scan and the patient was qualified for emergency surgery. Laboratory tests carried out 3 hours from dabigatran intake revealed activated partial thromboplastin time (APTT) of $29.7 \mathrm{sec}$ (normal range: 24-35) and glomerular filtration rate of $66 \mathrm{~mL} / \mathrm{min}$. It was suspected that the patient did not swallow the tablet. The administration of idarucizumab (Praxbind ${ }^{\circledR}$, Boehringer Ingelheim), the specific reversal agent for dabigatran, was considered before and during the surgery. Adequacy of intraoperative hemostasis was declared by the operating surgeon. Surprisingly, the post-operative lab results at 1500 were as follows: APTT $79.2 \mathrm{sec}$, thrombin time (TT) $>240 \mathrm{sec}$ (normal range: 14-21) and the dabigatran plasma concentration $207 \mathrm{ng} / \mathrm{mL}$. The administration of the idarucizumab was considered once more. Finally the antidote was not administered because there were no complications during the operation, no signs of postoperative bleeding and the patient was in stable condition.

On the following day the patient was stable, conscious, with good verbal contact, dabigatran plasma concentration was $128 \mathrm{ng} / \mathrm{mL}$, APTT $48.4 \mathrm{sec}$, TT 98 sec. Two days after operation the dabigatran plasma concentration was $46 \mathrm{ng} / \mathrm{mL}$, APTT $48.5 \mathrm{sec}$, TT 69.1 sec. On the $5^{\text {th }}$ postoperative day abdominal symptoms recurred. Computed tomography showed superior mesenteric artery embolism and embolectomy was successfully performed. After 20 days of hospitalization the patient was discharged to the Rehabilitation Department in stable condition. Clinical course of the patient is shown in Figure 1. 


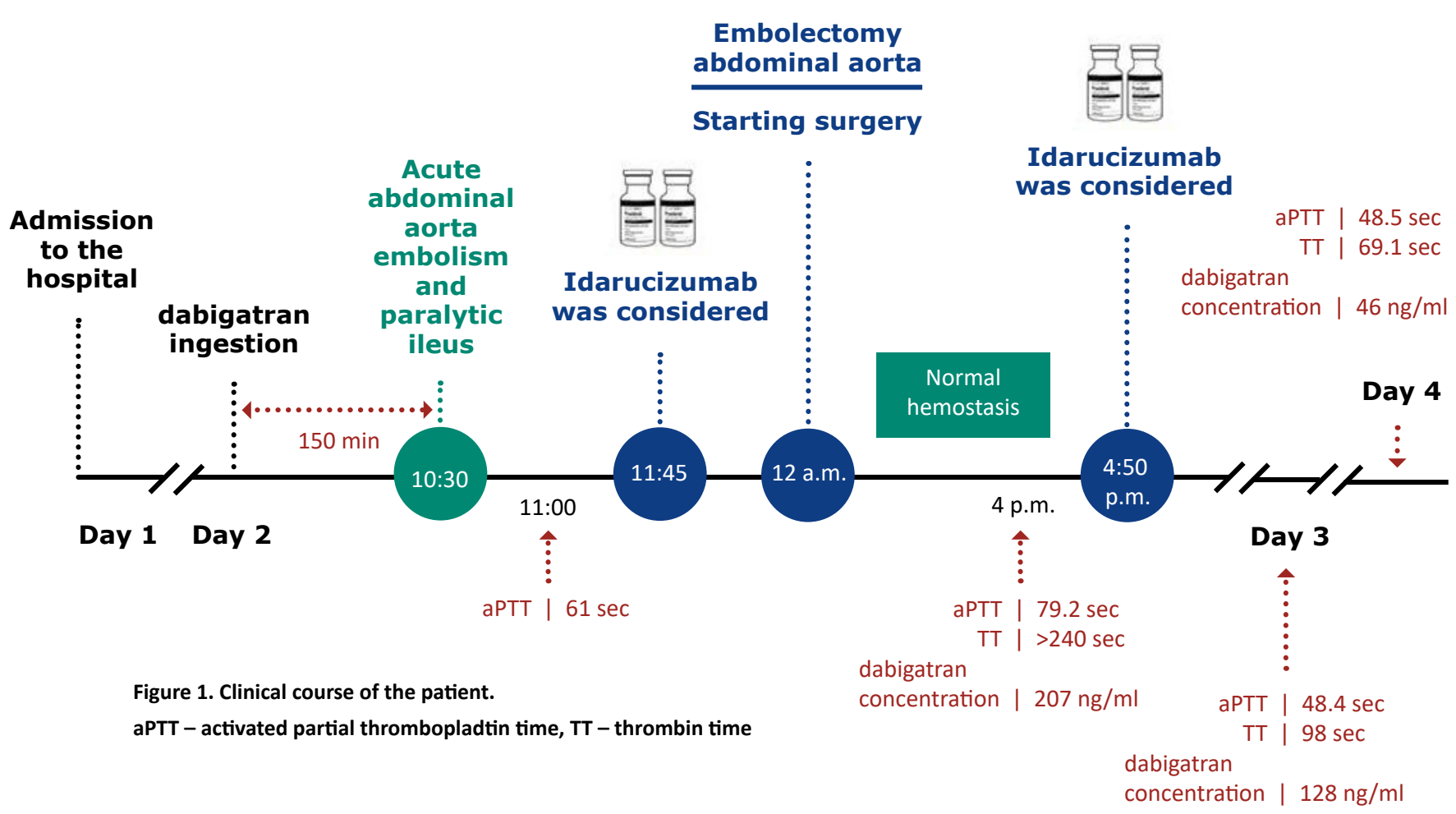

Discussion

Shortly after starting treatment with dabigatran, a 79-year-old woman presented to the university hospital with abdominal discomfort. She was to be operated few hours after dabigatran ingestion. Surprisingly APTT was in middle of the normal range. In fact, during the initial part of the operation there was relatively efficacious coagulation according to the surgeon's assessment. The decision about idarucizumab administration was difficult but eventually reversal agent was not given.

Significant anticoagulant activity, however, reoccurred 5 hours after the operation, and a dabigatran plasma concentration of $207 \mathrm{ng} / \mathrm{mL}$ was measured 8 hours after the last ingestion of the drug. Idarucizumab was considered again, but based on the patient's improving condition and no signs of bleeding it was decided against reversal agent. The decision to withhold idarucizumab at that time point is a matter of debate. We are aware that some practicing clinicians would administer idarucizumab to avoid possible serious bleeding complications.

In our opinion this is a clinically important case. The dabigatran plasma concentration of $128 \mathrm{ng} / \mathrm{mL}$ obtained on day 2 is an interesting finding. In contrast, the literature describes median (10th-90th percentile) steady-state peak plasma concentrations of 133 (52-275) and $184(74-383) \mathrm{ng} / \mathrm{mL}$ in patients with $\mathrm{AF}$ taking dabigatran at doses of 110 or $150 \mathrm{mg}$ twice daily, respectively [5-6]. Thus, our patient had a therapeutic

dabigatran plasma concentration about two half-lives after the last ingestion. The normal bioavailability of its prodrug (dabigatran etexilate) is 6-7\% [7-8]. Thus, it is conceivable that the patient's paralytic ileus caused altered absorption, resulting in increased exposure to the anticoagulant a few hours later [8]. Our patient's coagulation markers such as APTT and TT corresponded with dabigatran plasma concentrations. We found one similar case in the literature; a patient with rectal perforation and peritonitis, who underwent emergency surgery complicated by severe bleeding stopped by idarucizumab and with postponed absorption of dabigatran [9].

Also according to the summary of product characteristics (SmPCs), dabigatran reaches maximum concentration in the plasma within 6 hours instead of 2 hours following administration in a postoperative period due to the influence of factors such as general anesthesia, gastrointestinal paresis, and surgical effects, regardless of the form in which it is orally administered [9-10]. Although these mechanisms remain not fully explained and at this time are speculative, alterations in absorption in conditions of ileus or acute peritonitis may potentially explain this phenomenon.

Dabigatran is considered as a safe drug according to both randomized trials and registries with low rate of major bleeding and a significant reduction of intracranial bleeding as compared to warfarin. 


\section{Conclusions}

We conclude that exposure to dabigatran may occur later in patients with acute ileus than it can be expected in normal situation due to alterations in absorption. Such patients should be carefully monitored for a significant rebound in anticoagulant activity. The use of specific reversal agent for dabigatran in similar cases should be carefully and individually considered.

\section{Funding}

No funding has been received for preparation of this manuscript.

\section{Conflict of interests}

A. Tomaszuk-Kazberuk has received honoraria from Boehringer Ingelheim for lectures and consultations. B. Korbel is a medical manager at Boehringer Ingelheim.

\section{References}

1. Pollack Jr C V, Reilly PA, Eikelboom J, Glund S, Verhamme P, Bernstein RA, et al. Idarucizumab for dabigatran reversal. N Engl J Med. 2015;373(6):511-20.

2. Pruszczyk P, Tomaszuk-Kazberuk A, Słowik A, Drwiła R, Rydzewska G, Filipiak KJ, et al. Management of bleeding or urgent interventions in patients treated with direct oral anticoagulants: 2017 recommendations for Poland. Pol Arch Intern Med. 2017;127(5):343-51.

3. Tomaszuk-Kazberuk A, Łopatowska P, Młodawska E, van Ryn J, Reilly PA, Pollack C V. Successful use of idarucizumab as a reversal agent for dabigatran in a patient with acute dissected aortic aneurysm. Pol Arch Intern Med. 2017;127(1):68-70.

4. Eikelboom JW, Quinlan DJ, van Ryn J, Weitz JI. Idarucizumab: the antidote for reversal of dabigatran. Circulation. 2015;132(25):2412-22.

5. Lang T, Bauters A, Braun SL, Pötzsch B, von Pape K-W, Kolde H-J, et al. Multi-centre investigation on reference ranges for ROTEM thromboelastometry. Blood Coagul fibrinolysis. 2005;16(4):301-10.

6. Reilly PA, Lehr T, Haertter S, Connolly SJ, Yusuf S, Eikelboom JW, et al. The effect of dabigatran plasma concentrations and patient characteristics on the frequency of ischemic stroke and major bleeding in atrial fibrillation patients: the RE-LY Trial (Randomized Evaluation of Long-Term Anticoagulation Therapy). J Am Coll Cardiol. 2014;63(4):321-8.

7. Hankey GJ, Eikelboom JW. Dabigatran etexilate: a new oral thrombin inhibitor. Circulation. 2011;123(13):1436-50.

8. Thorborg C, Horn E-P, Mofid H, Langer F. Reversal by the specific antidote, idarucizumab, of elevated dabigatran exposure in a patient with rectal perforation and paralytic ileus. Br J Anaesth. 2016;117(3):407-9.

9. The summary of product characteristics (SmPCs): Pradaxa $150 \mathrm{mg}$. 